\title{
Automatic Discovery of Basic Motion Classification Rules
}

\author{
Satoshi Hori ${ }^{1}$, Mizuho Sasaki ${ }^{1}$, and Hirokazu Taki ${ }^{2}$ \\ ${ }^{1}$ Monotsukuri Institute of Technologists \\ 333 Maeya, Gyoda 361-0038 Japan \\ hori@iot.ac.jp \\ http://www.iot.ac.jp/manu/HORI.html \\ ${ }^{2}$ Wakayama University \\ 930 Sakaedani, Wakayama 640-8510 Japan
}

\begin{abstract}
There is a keen demand for a method of sharing better work practices in a factory because better work practices are the key to improving productivity. We have developed a system that can measure a worker's motion and automatically generate a manual that describes his movements. This system employs motion study as used in Industrial Engineering to identify the important steps in a job, and it has proven to be effective especially in the fields of factory machine operation and maintenance. However, work procedures often include unique basic motions. The determination of basic motions and the creation of an algorithm that can classify these basic motions are time consuming and complex tasks. Therefore we have employed the $\mathrm{C} 4.5$ algorithm to discover rules that classify the basic motions. Experimental results prove that our method can successfully discover rules for various work procedures.
\end{abstract}

\section{Introduction}

Firstly we outline why better work practices need to be recorded and shared. The objective of automatic discovery of basic motion classification rules is then described after a brief introduction to our manual generation system.

\subsection{Background: Better Work Practices Need to Be Shared}

Better work practices contribute greatly to higher productivity in factories. Skilled workers have better work practices because they have learned through experience in the workplace. For example, a service technician or worker moves his arms and legs while he checks and repairs machinery. He walks, pushes switches, and looks at meters. The behavior of an experienced technician is more efficient than that of a novice, and we can learn a lot by observing the work practices of a well-trained and experienced worker. Efficient work processes need to be documented in manual form and shared with others. Good operation manuals improve work productivity. There is a keen demand for manual generation systems because documenting work processes is a time-consuming task. This is the motivation of our research.

\subsection{Objectives: To Automatically Discover Basic Motions}

We have developed a system that records a worker's actions in order to generate a manual. Our system employs motion study [1] used in Industrial Engineering (I.E.) to 
identify important events in a work procedure. Why is the identification of important events required? An item of work may take from 30 minutes up to several hours to complete. Hence, it is necessary to pick up several important events in the work procedure in order to teach novices the key steps in the work.

Motion study shows that a work process is a series of basic motions. Most work is done using the two hands, and all manual work consists of relatively few fundamental motions which are performed over and over again. "Get" or "pick-up" and "place" or "put-down" are two of the most frequently used groups of motions in manualassembly work.

Motion study has a history going back almost one hundred years, and it is still used in many production lines. However, basic motion recognition still depends on human I.E. engineers. On top of this, a work procedure often includes unique basic motions. It is a time-consuming and complex task to determine basic motions and create an algorithm that can classify the basic motions. Therefore we have employed the C4.5 algorithm to discover rules that classify the basic motions. Some experimental results prove our method can successfully discover the rules for various work procedures.

\section{Skill Intelligence and Motion Study}

Experienced workers' skill is the key to improved productivity in a manufacturing facility, and a method for sharing skill intelligence has been in demand. This section defines skill intelligence and briefly introduces motion study. Our manual generation system is also explained.

\subsection{Definition from the Hierarchical Paradigm of Robotic Control}

Knowledge and/or intelligence can be found not only in the form of language but also in the behaviors and skills of living creatures. With a motion capture system, ubiquitous sensors, and other electronic devices, we can electronically observe and record human motions. The skill intelligence elicited from such data will be useful if it is shared among novice workers.

The term 'skill' includes a wide range of meanings. When a very fast ball is pitched or a violin is played, skill is required. In this case, 'skill' involves the control of muscles. On a factory floor, manufacturing machinery has to be appropriately operated and maintained. In this case, 'skill' includes knowledge and experience.

Robotics provides a good domain for recognizing skills on a factory floor as compared to, for example, the skill required to play a violin. The hierarchical paradigm [2] is one method for organizing intelligence in mainstream robotics. This paradigm, as shown in Figure 1, is defined by the relationship between the three primitives (SENSE, PLAN, ACT). Considering a walking robot, first the robot senses the world, the robot plans all the directives needed to reach a goal, and the robot acts to carry out the first directive.

Human skills exist in Sense, Plan, and Act modules. Some experienced workers can sense $1 \mu m$ noise on a steel sheet. On the other hand, the skill intelligence that 


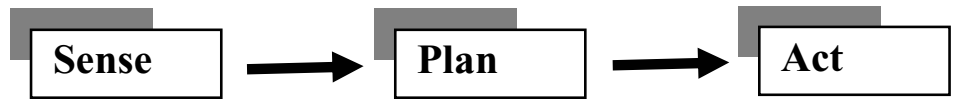

Fig. 1. Hierarchical Paradigm of Robot Controller

we will be storing and sharing is 'skill' in the Plan module. An experienced worker can make an appropriate plan for fabrication and repair because he has a lot of background knowledge and experience. This task can be realized as a planning and classification task in artificial intelligence. This skill intelligence is rarely documented in manual form. Therefore a system that can observe worker's actions and generate a manual automatically is required.

\subsection{Industrial Engineering (I.E.) Motion Study}

Industrial Engineering (I.E.) provides a strategy for finding a preferred method of performing work, which is referred to as Motion Study [1]. Frank and Lillian Gilbreth did pioneering research in this area and established the field of Motion Study in the beginning of the $20^{\text {th }}$ century. Glibreth noticed that most work is done with two hands, and all manual work consists of relatively few fundamental motions that are performed over and over again. "Get" or "pick up", and "place" or "put down" are two of the most frequently used groups of motions in a production line. Gilbreth developed subdivisions or events, which he considered common to all kinds of manual work. He coined the word therblig to refer to any of the seventeen elementary subdivisions.

$>$ Motion sensing, motion labeling, and work recognition: an I.E. engineer observes what a worker is doing on a production line. He understands the worker's movements and writes them down as a series of basic motions using therblig symbols. He then establishes how the work is done. Table 1 describes an example.

$>\quad$ Use of observed motion: The record of basic motions is analyzed and used for designing a better work procedure.

The I.E. Motion Study is expensive because it manually analyzes the worker's movement.

Table 1. Example of Motion Study: Task of "signing a paper"

\begin{tabular}{|l|l|}
\hline \multicolumn{1}{|c|}{ Therblig } & \multicolumn{1}{c|}{ Description of Motion } \\
\hline 1. TE: Transport Empty & Reach for pen. \\
\hline 2. G: Grasp & Take hold of pen. \\
\hline 3. TL: Transport Loaded & Carry pen to paper. \\
\hline 4. P: Position & Position pen on paper for writing. \\
\hline 5. U: Use & Sign the paper \\
\hline 6. TL: Transport Loaded & Return pen. \\
\hline
\end{tabular}




\subsection{Proposed System}

Figure 2 depicts the outline of our proposed system. The system consists of two subsystems: (1) a wearable sensor system that observes the body movement and vision range of a worker, (2) a manual-generator that generates a manual from the motion data recorded by the wearable sensor system. We employed IC accelerometers to measure a worker's motion. A camera is used to record what the worker sees while doing a job.

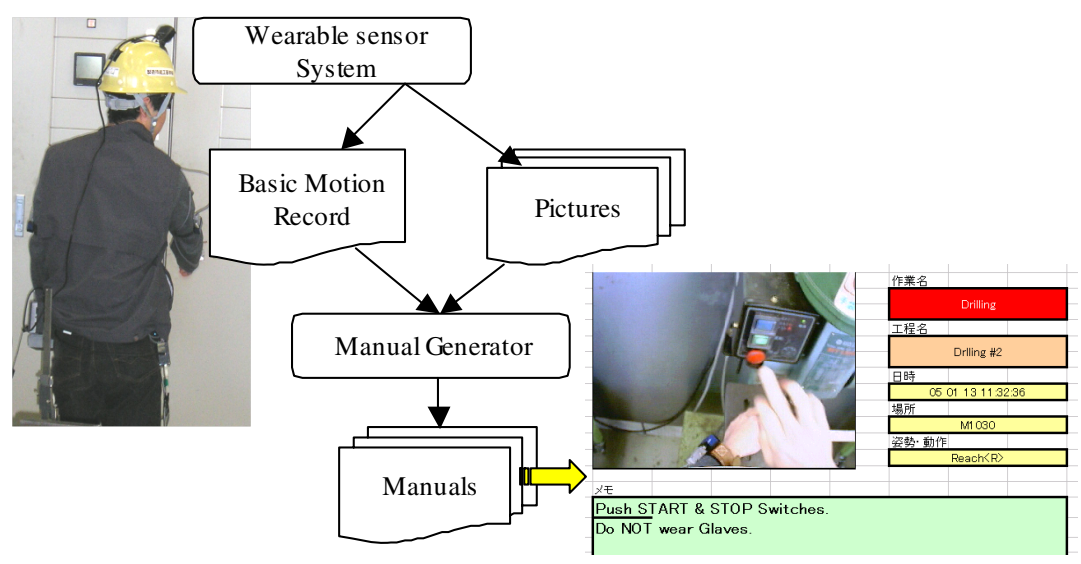

Fig. 2. Our Manual-Generation System

\subsubsection{Motion Observation with IC Accelerometers}

We employed IC accelerometers to measure motion [2]. The sensors are attached to the arms and legs of a service technician. This section describes IC accelerometers and their signal processing. Analog Device's ADXL202 is employed as an accelerometer. The ADXL202 is a low-cost, low-power, complete 2-axis accelerometer sensor with a measurement range of $\pm 2 \mathrm{~g}$. The sensors attached to a right arm are shown in Figure 3. Figure 4 demonstrates how "reach" motion is detected intuitively from the output of a wrist sensor. "Reach" motion often occurs when a technician operates switches. The right hand is first extended close to the right leg. In the "reach" motion, the hand is raised and lowered.

To detect a basic motion, the output signal is processed as follows:

Step-1. Measure raw X, Y acceleration data with a $100 \mathrm{msec}$ sampling rate.

Step-2. Eliminate noise by taking the moving average of each of five samples.

Step-3. Data labeling: Classify each data item into 3 bins labeled $-1,0$, and 1 .

This data set is described as a gravity pattern.

Step-4. Motion labeling: Prepare a table that maps a gravity pattern change to a basic motion. As an example, when an arm's gravity pattern changes from 0101 to 1010, we determine that "reach" motion is occurring (see Figure 4). 


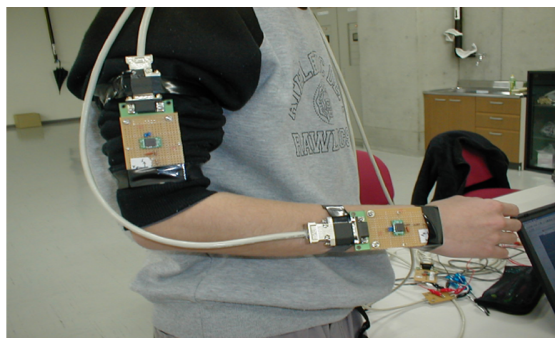

Fig. 3. Accelerometers on Right Arm

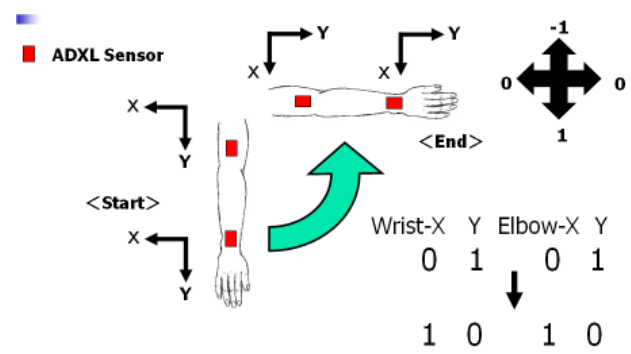

Fig. 4. Wrist Accelerometer's Output for Reach Motion

\section{Basic Motion Discovery}

A basic motion is a fundamental motion that occurs over and over again while performing a job. When a basic motion is observed, we recognize it as an important event worth recording. Our method can generate a set of rules that discover basic motions. The rules are generated from a set of supervised learning data with the C4.5 algorithm [3].

\subsection{C4.5 Algorithm}

The algorithm C4.5 uses top-down induction of decision trees. Given a set of classified examples, a decision tree is induced, biased by the information gain measure, which heuristically leads to small decision trees. These trees can be easily transformed into a set of production rules. Learning data, a set of examples, are given in attribute-value representation. The set of possible classes is finite.

Table 2 shows an example of classes. Part of the learning data is shown in Table 3. The goal of this example is to discover rules that decide whether people play golf, if weather outlook, temperature, etc., are given. Figure 5 lists the generated rules.

Table 2. Attributes and Values for Golf Problem

\begin{tabular}{|l|l|}
\hline \multicolumn{1}{|c|}{ Attributes } & \multicolumn{1}{c|}{ Value } \\
\hline Outlook & sunny, overcast, rain \\
Temperature & cool, mild, hot \\
Humidity & high, normal \\
Windy & true, false \\
\hline
\end{tabular}

Table 3. Supervised Learning Data for Golf Problem

\begin{tabular}{|c|c|c|c|c|c|}
\hline \multirow{2}{*}{ No. } & \multicolumn{4}{|c|}{ Attributes } & \multirow{2}{*}{ Class } \\
\cline { 2 - 5 } & Outlook & Temperature & Humidity & Windy & \\
\hline 1 & sunny & hot & high & false & no play \\
2 & sunny & hot & high & true & no play \\
3 & overcast & hot & high & false & play \\
$:$ & $:$ & $:$ & $:$ & $:$ & $:$ \\
14 & rain & mild & high & true & no play \\
\hline
\end{tabular}




\begin{tabular}{|cc|}
\hline Rule 1: & IF Outlook = overcast $\quad$ THEN play golf. \\
Rule 2: & IF Outlook = sunny AND Humidity = high \\
& THEN do not play golf \\
& $:$ \\
\hline
\end{tabular}

Fig. 5. Generated Rules

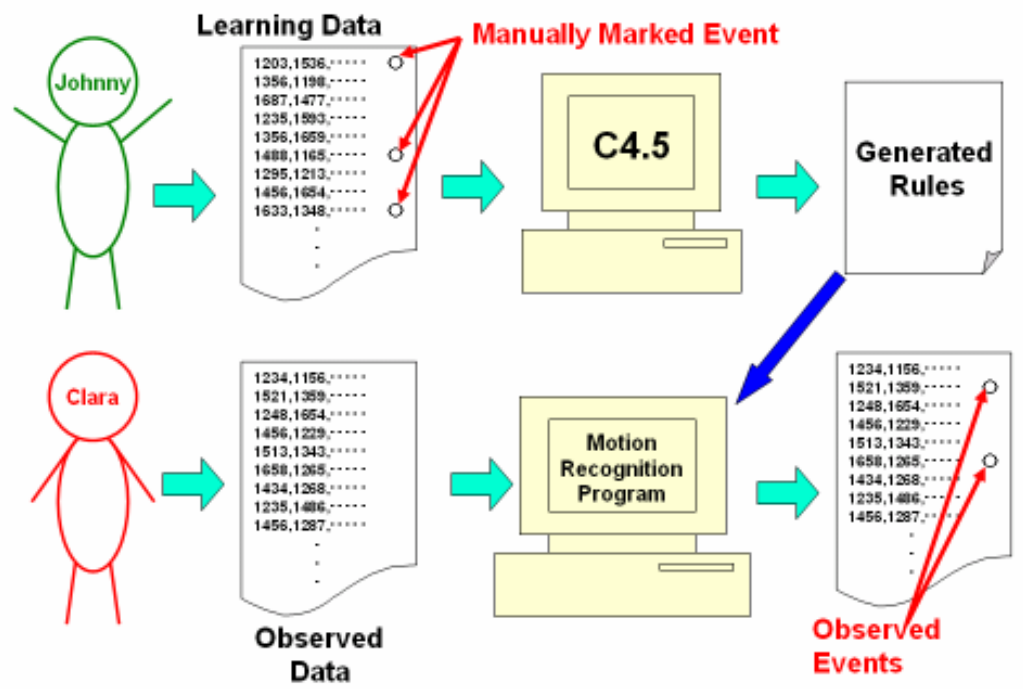

Fig. 6. Outline of Basic Motion Discovery

\subsection{Outline of Classification Rule Discovery}

The framework of rule discovery and basic motion detection is described in Figure 6 . This procedure consists of two major parts, (1) Rule discovery, and (2) Basic motion classification using the rules discovered.

(1) Rule discovery

Step-1. Prepare supervised learning data.

Human motion data, Sensor outputs, are recorded while Johnny performs a certain target job. Important events are manually marked. Thus we obtain the supervised learning data.

Example: A mouse button is pushed when Johnny throws a dart, so this throwing event is marked on the data file.

Step-2. Prepare the data file.

Details of this preparation are described in the next section.

Step-3. Feed this data file to the C4.5 program. C4.5 generates the classification rules. 
Example of generated rules:

IF $R W Y<-90[$ deg] THEN dart throw occurred.

(2) Basic motion classification

Step-1. The rules discovered above are implemented in a recognition program.

Step-2. Clara's motion is recorded while Clara performs the same job.

Step-3. The classification program determines what basic motion occurred using the rules.

Example: Events in which Clara threw a dart are marked in the output file of the recognition program.

\subsection{Learning Data Preparation}

This section describes how to prepare learning data from the observed motion data. We take dart-throwing as an example. In the case of dart-throwing, we want to identify events involved in throwing a dart.

The learning data is depicted in Figure 7. Two sensors are attached, one to the wrist and the other to the elbow of the right arm. Observed data consist of the voltage output by the sensor. RWX denotes the voltage of the Right arm's Wrist X-axis output. REY denotes the voltage of the Right arm's Elbow Y-axis output.

The sensor data are sampled with a $10 \mathrm{~Hz}$ sampling frequency. Each record in the observed data represents a right arm pose carried out every 0.1 second. The dartthrower pushes a button when he throws a dart, and the "Throw" label is added to a record, as shown in Figure 7(B).

The records in a 0.5 -second interval are assembled to give a record of the learning data, as shown in Figure 7(C). One record in Figure 7(C) denotes a move of 0.5 seconds. C4.5 processes this supervised learning data and generates rules that distinguish the dart-throwing and other movements.

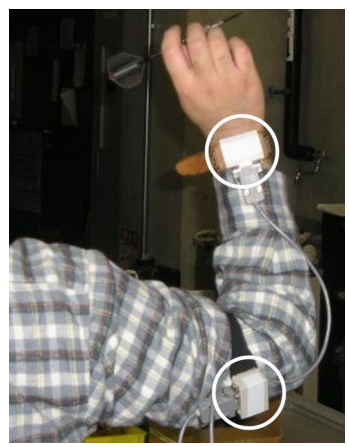

\begin{tabular}{|l|l|l|l|}
\hline RWX & RWY & REX & REY \\
& & \\
\end{tabular}

(a) Sensor Data Set at t [sec]

Added Classes
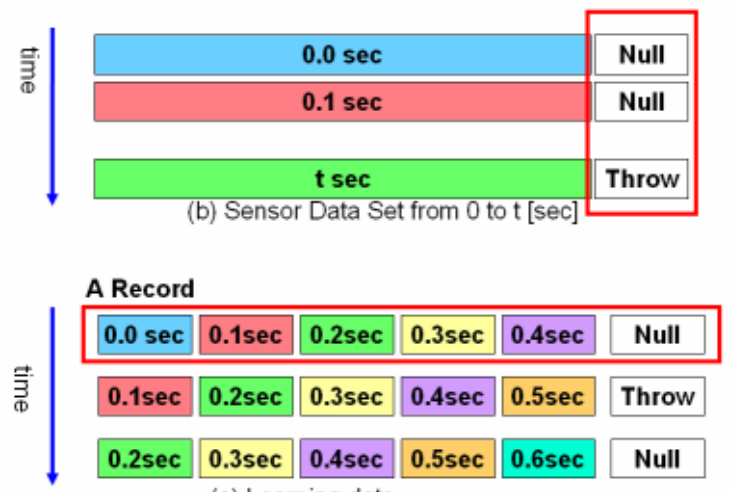

(c) Learning data

Fig. 2. Dart Throwing and Learning Data 


\subsection{Experimental Results}

We conducted experiments for two types of motion, dart-throwing and confirmationby-pointing, in order to evaluate how effectively the generated rules identify important events in the stream of motion.

- Dart-throwing

Firstly, we generated rules for the dart-throwing task. 10 dart-throwing motions were recorded as supervised-learning data. $\mathrm{C} 4.5$ then generated the following rules that recognize dart-throwing motion as distinct from other motion.

Rule-1: IF RWY <-90[deg] THEN dart-throw occurred.

Rule-2: IF RWY at 0.2[sec] > 90[deg] AND RWY at 0.4 [sec] $<60$

THEN dart-throw occurred.

The rules successfully classified all 55 dart-throwing events. No false classifications occurred. 23 false accepts did occur but these were the motions of retrieving darts from a target. The reason the program misclassified these motions is because this retrieving-darts motion was not included in the learning data.

Table 4. Dart-throwing

\begin{tabular}{|l|l|l|l|}
\hline No. of Events & Correct & FA & FR \\
\hline 55 & 55 & 23 & 0 \\
\hline
\end{tabular}

FA: False Accept, FR: False Reject.

- Confirmation-by-pointing

Secondly, Confirmation-by-pointing was tested. Assumed work is as follows: A participant in the experiment counts red boxes in a warehouse. He ensures that all red boxes are counted by pointing at them one by one. His right arm moves in a manner similar to the "Reach" motion when he performs this confirmation-by-pointing motion.

We observed ten confirmation-by-pointing motions performed by the person. C4.5 generated a rule as follows:

\section{Rule-1: IF REY at 0.2[sec] < 70[deg] AND RWY at 0.2 [sec] > 40[deg] $A N D$ REY at 0.4[sec] $<70$ [deg] THEN Confirmation-by-pointing occurred.}

Using this rule, the recognition program classifies the confirmation-by-pointing motions performed by another person. Table 5 shows the result. The participant performed the confirmation-by-pointing eight times. Seven out of the eight motions were successfully classified. One motion was missed.

Table 5. Confirmation-by-pointing

\begin{tabular}{|l|l|l|l|}
\hline No. of Events & Correct & FA & FR \\
\hline 8 & 7 & 0 & 1 \\
\hline
\end{tabular}

FA: False Accept, FR: False Reject. 


\section{Conclusion}

There is a keen demand for a method of sharing better work practices in a factory because better work practices are the key to improving productivity. To analyze work practices, we need to recognize and record a human worker's motion. Motion study used in Industrial Engineering provides a good method for analyzing and designing better work practices. However we also need to develop algorithms to classify basic motions for the motion study. In this paper, we employed the $\mathrm{C} 4.5$ algorithm to automatically generate classification rules for basic motions. The experimental results of section 3.4 prove the effectiveness of our method.

\section{References}

[1] Barnes, "Motion and Time Study", John Wiley \& Sons, Inc., (1968).

[2] Robin R. Murphy, "Introduction to AI Robotics", MIT Press, (2000).

[3] J.R. Quinlan, "C4.5 an Induction System", Academic Press ().

[4] J. R. Quinlan: "Decision Trees and Decision making", IEEE Trans. on Systems, Man and Cybernetics, Vol.20,No.2, pp.339-346 (1990).

[5] S.Hori, K.Hirose, H.Taki, "Acquiring After-Sales Knowledge from Human Motions", KES2004, LNAI3214, pp.188-194 (2004).

[6] T. Nakata, "Automatic Generation of Expressive Body Movement Based on CohenKestenberg Lifelike Motion Stereotypes," Journal of Advanced Computational Intelligence and Intelligent Informatics, Vol.7 No.2, pp.124-129, (2003).

[7] Song, Goncalves, Perona, : "Unsupervised learning of human motion", IEEE Transactions on Pattern Analysis and Machine Intelligence, Vol.25, Iss.7, pp.814-827 (2003). 